\title{
Designing digital technology tasks for the development of functional thinking
}

\author{
Stephan Michael Günster ${ }^{1} \cdot$ Hans-Georg Weigand ${ }^{1}[\mathbb{D}$
}

Accepted: 25 June 2020 / Published online: 15 July 2020

(c) The Author(s) 2020

\begin{abstract}
In this paper we introduce a theoretical framework concerned with fostering functional thinking in Grade 8 students by utilizing digital technologies. This framework is meant to be used to guide the systematic variation of tasks for implementation in the classroom while using digital technologies. Examples of problems and tasks illustrate this process. Additionally, results of an empirical investigation with Grade 8 students, which focusses on the students' skills with digital technologies, how they utilize these tools when engaging with the developed tasks, and how they influence their functional thinking, are presented. The research aim is to investigate in which way tasks designed according to the theoretical framework could promote functional thinking while using digital technologies in the sense of the operative principle. The results show that the developed framework-Function-Operation-Matrix-is a sound basis for initiating students' actions in the sense of the operative principle, to foster the development of functional thinking in its three aspects, namely, assignment, co-variation and object, and that digital technologies can support this process in a meaningful way.
\end{abstract}

Keywords Functional thinking $\cdot$ Operative principle $\cdot$ Task design $\cdot$ Digital technologies $\cdot$ Empirical investigation

\section{Introduction}

Functions are a common thread that runs through all of mathematics education and acts as a guideline throughout the whole mathematical curriculum (e.g. Core-PlusMathematics Project 2015). Through exposure to functions, students acquire a variety of topic-related and universal mathematical skills. They recognize and describe functional relationships in different situations, analyze, interpret and compare various representations of functions, characterize functions by their properties and solve realistic problems with the help of functions.

The development of the function concept in school is based on a wide spectrum of phenomena and depictions. Learners develop an idea of the function concept in relation to different situations and representations and come to understand how these are interwoven with the technical

Hans-Georg Weigand

weigand@mathematik.uni-wuerzburg.de

Stephan Michael Günster

stephan.guenster@uni-wuerzburg.de

1 Julius-Maximilians-University, Würzburg, Germany aspects of the concept. Hence, the concept of function develops on various abstract levels.

While developing mathematical concepts in the classroom, tasks play a very important role. They substantiate educational objectives and skills associated with understanding the concept. However, the term 'task' covers a wide range of applications in mathematics education. A task can vary from a single question asked by a teacher, over a system of exercises, to a whole project or to "rich learning tasks" (Flewelling and Higginson 2003), as Leuders (2015) observes, "A (mathematical) problem outlines a (mathematical) situation which encourages the learner to a (mathematical) engagement with this situation" (p. 435).

In accordance with this very general definition and the wide spectrum of applications, task design is versatile (Watson and Ohtani 2015). It ranges from the construction of tasks and the corresponding principles of design over composition in regard to content in different learning environments to an emphasis on connections between different mathematical proficiencies and their use in regard to assessment. The main research aim presented in this paper is to develop a theoretical framework for task design in order to promote functional thinking while using digital technologies, in the sense of the operative principle. 


\section{Literature survey}

\subsection{Task design and digital technologies}

This paper focuses on task design with the aid of, and in conjunction with, digital technologies. Mathematical tools enable us to create, to operate with, and to transform mathematical objects. Computers, and especially programs such as spreadsheets, dynamic geometry systems (DGS) and computer algebra systems (CAS), are digital mathematical tools. In 2010, the 17th ICMI study Mathematics Education and Technology: Rethinking the Terrain (Hoyles and Lagrange 2010) was published, revisiting the title of the very first ICMI study The Influence of Computers and Informatics on Mathematics and its Teaching (Churchhouse 1986). Given the great enthusiasm 20 years earlier for the new possibilities that computers and technology might open to mathematics and mathematics education, the 2010 study gave a disappointing account of the current situation concerning the dissemination of technology. Despite a high number of research studies and accounts of classroom practices, the use of technologies in mathematics education and the impact on curriculum and assessment was still limited.

Traglová et al. (2018) documented and evaluated the research in mathematics education in the last 20 years concerning the impact of digital technologies on mathematical learning and teaching as reflected in the CERME conferences starting in 1999. Especially two findings came out of this retrospective view: One was the importance of task and the other the importance of a theoretical framework, further, "...it was also apparent that the reflective use of tools in the learning process needed theoretical frameworks specific to the tool and mathematical content, for which the Instrumental Approach emerged as a central theoretical framework" (Traglová et al. 2018, p. 145).

The design of digital mathematical tasks has recently received particular attention, as a sub-theme of the 22nd ICMI Study Task Design in Mathematics Education (Watson and Ohtani 2015) and within a dedicated volume of the Mathematics in the Digital Era book series (Leung and Baccaglini-Frank 2017). At the very first meeting of the relevant thematic working group of the CERME conferences, Gutiérrez et al. (1999), highlighted the main point:

One of the key issues for teachers is how to design tasks based on tools or technologies in which real questions for the learner emerge from the use of the tool, in which the tool is relevant and gives a new dimension to the task. (p. 187)

Task design has two main aspects on which the focus may be placed:
1. Development of innovative technological tasks that provide access to traditional mathematical knowledge and activity.

2. Development of innovative technological tasks that might lead to new forms of mathematical knowledge and activity.

Leung and Bolite-Frant (2015) also noted, "A toolbased task is seen as a teacher/researcher design aiming to be a thing to do or act on in order for students to activate an interactive tool-based environment where teacher, students, and resources mutually enrich each other in producing mathematical experiences" (p. 192). Thus, a digital-tool-based task has to be seen in relation to teaching, learning, mathematical knowledge and the mediation of digital technologies (Traglová et al. 2018).

Our study focused on the design of tasks in order to develop functional thinking with the aid of digital-toolbased tasks. We especially see the interrelationship of tools and learners, how the tools influence the thinking and operating of the learner, and vice versa, how learning forms and changes the tool. This is usually called the "instrumental genesis", "instrumental approach" or "instrumental orchestration" (e.g., Trouche 2004; Drijvers et al. 2010).

\subsection{Functional thinking}

Since the beginning of the twentieth century and the Merano Reform (1905), essentially initiated by Felix Klein (1849-1925), the concept of functional thinking in mathematics education has been widely discussed (Krüger 2019). For Felix Klein functional thinking was a guiding principle for teaching mathematics in order to unify different areas of school mathematics. However, the concept of functional thinking was quite open and not clearly defined by Felix Klein; consequently, the school reality in the up-coming years of the twentieth century was that it was reduced to working with elementary functions in algebra and calculus. At the end of the twentieth century, there was renewed interest in the original ideas of Felix Klein, especially in clarifying the concept of functional thinking. One example is that of Vollrath's (1989) definition, "Functional thinking is a way of thinking which is typical for working with functions" ( $p$. 85). While this definition looks — at first sight—strange or even contradictory, it transfers the unclear concept of functional thinking to a tangible mathematical aspect: working with functions.

Henceforth, we distinguish three characteristics of working with functions (e.g., Doorman et al. 2012; Dubinsky and Harel 1992; Vollrath 1989) by adopting Vollrath's three aspects: 
- Assignment aspect: a function creates a relation between two variables. For a given input, an output is calculated. The level of understanding can be determined by how relations are recognized and worked with in different forms of representation.

- Co-variation aspect: a function describes how changes in the independent variable affect the dependent variable. Typical activities for this aspect are to plan, execute or analyse variations of the independent variable and the resulting covariations.

- Object aspect: a function can be seen as a whole and therefore be dealt with as a mathematical object. This means that attributes can be used to describe a function as a whole (e.g., periodicity, monotonicity) but can also be derived from it. Furthermore, one can treat functions like mathematical objects in their own right that can be operated on (e.g., by adding or substituting).

All aspects can be visualized and analyzed if typical representations are seen under a particular perspective. However, some representations are more suitable for certain aspects than others. For example, graphs offer the opportunity to display a wide range of pairs of values. This is helpful in viewing the function as a whole (object aspect) whereas a table shows only a limited number of values. A learner should therefore be able not only to work with, but also to choose, a suitable representation flexibly (Acevedo Nistal et al. 2012).

\subsection{The operative principle}

One possibility for developing functional thinking is the functional observation of operations and examining the changes caused by these operations (Vollrath 1989, p. 21). While Vollrath used the term operation in this context in the sense of a mathematical operation such as multiplication or addition, different theoretical frameworks concerned with the learning process utilize it with varying meanings (e.g., Arnon et al. 2014; Giest and Lompscher 2006; Piaget 1967; Vygotsky 1978; Wittmann 1985). Therefore, we now define the terms important for the design principles that we present in this paper.

According to Piaget (1967), understanding of a concept begins with actions (see also, e.g., Dubinsky and Harel 1992). An action is a repeatable manipulation of objects and hence cannot be viewed on its own but must be considered in relation to the objects, as well as the subject who is performing the action. If these actions are repeated and reflected upon, they can be interiorized as flexible mental processes (cf. Arnon et. al 2014; Piaget 1967), so-called operations. Thus, the difference between actions and operations is that learners can perform the actions directly in a chosen form of representation and observe the effects there, while the operations are performed purely mentally.

Just as with actions, operations must be viewed as acting on objects and the effect of changes on their properties and relations needs to be evaluated. This goes along with Wittmann (1985), who commented, "to comprehend objects means to investigate how they were constructed and how they behave when operations are applied on them" (p. 9, translated by the authors). We call this the operative process: it describes the relationship between the subject and the object by manipulation through actions or operations and the evaluation and reflection of the resulting changes. On the one hand, this leads to the interiorization of actions into operations. On the other hand, the subject-the learnergains knowledge about the objects as well as about the actions or operations.

In order to use this approach in the learning process, learners need opportunities to be engaged in the operative process, placed into situations in which they will ask characteristic questions like "What happens with ... if ..." in the sense of an action consequence principle (cf. Burrill 2017). Therefore, one could adapt tasks by varying the involved objects, actions and operations or the relations between them to find interesting effects or invariants. Such an approach is referred to as the operative principle (Wittmann 1985). In summary, two basic principles for the application of the operative principle can be identified: First, tasks should be varied systematically and the changes through these variations need to be evaluated. Second, the impact on the observed objects by actions or operations in accordance with the question "What happens with ... if ..." should be examined and reflected upon.

\subsection{Summary and research questions}

The operative principle is useful for developing functional thinking utilizing interactive dynamic representation via digital technologies for various reasons. Actions can be performed directly on the given objects, for example, by dragging points or using sliders, and the changes can be viewed accordingly. Thereby one can investigate invariances and variations directly through the given representation. In addition, actions become possible that would be very difficult to carry out with paper and pencil, such as shifting a function graph as a whole or adjusting parameters.

Relations between different variables can be studied by varying one variable in a certain context, for example in a geometric one, examining the changes caused to the dependent variable and interpreting them as a functional relationship. This can lead step by step, starting for example with the assignment aspect, to the further aspects of functional thinking. We illustrate this process by examples of tasks (Sect. 3).

In this paper, we address the following research questions: 
1. What is the nature of a theoretical framework that builds and uses the relationship between the concept of functional thinking and the operative principle for task design, and how can it be applied to develop interactive dynamic tasks?

2. In what way do students utilize digital technologies in the context of the developed tasks, and do they make use of the digital technologies as intended in the sense of the operative principle?

3. Which actions or operations do students exhibit in the context of the developed tasks that can be associated with the different aspects of functional thinking, and how does the operative process interact with and promote functional thinking?

While research question 1 is a theoretical one and answered in the following section, we conducted an empirical study to respond to questions 2 and 3 , which we then present.

\section{Own theoretical framework}

Developing functional thinking is the underlying goal of the approach presented in this paper. There exists a wellaccepted model for the concept of functional thinking with three characterizing aspects (see 2.2). We wanted to study the effect of using digital technologies in the sense of the operative principle. We identified two basic principles for the application of the operative principle: The variation of tasks and effects of these variations (see 2.3). To operationalize the variation of tasks we took an also quite established concept: a task can be divided into three phases (cf. Bruder 2000). First, there is the starting condition that describes the initial situation of the task, including the given values and information about a possible context. Secondly, to solve the task specific transformations are necessary which must be applied to the starting condition to get the desired-and third-target configuration. While the target configuration represents the result, a statement which is supposed to be proven or simply a wanted object, the transformations can have various forms. These could be as simple as one action which leads straight to the solution-the target configuration-but could also stand for multiple steps. There could also be different transformations, or ways of solving the tasks, that lead from one starting condition to the same target configuration. The operative principle can be seen concerning these three phases and their variation.

We see the relation between the concept of functional thinking and the operative principle as a two dimensional Function-Operation-Matrix (FOM) (see Fig. 1). One dimension arises from the concept of functional thinking with the three characteristic aspects. The second dimension describes

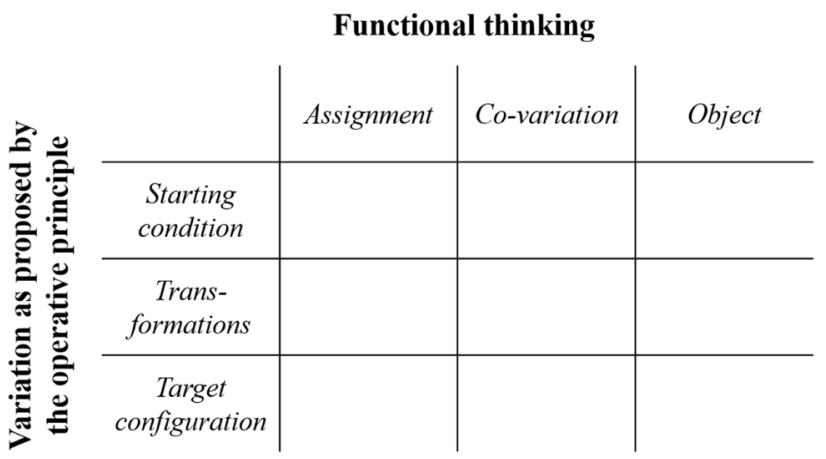

Fig. 1 The Function-Operation-Matrix (FOM): tasks are meant to be varied in accordance with the operative principle and with respect to the three characteristic aspects of functional thinking

the operative principle and its application to variation in the task by applying it to the three phases of a task. Thus, in order to design a task with regard to the FOM, one has to first think about when and which aspect of functional thinking is addressed. Furthermore, one has to consider which phase of the task indicates itself suitable for variation. Hence, the FOM is a guideline for task construction by analyzing and building a task following the nine cells of the FOM. For moving through the FOM when designing a task, two main principles can be deduced: First, one should move to the right in terms of the horizontal axis as this represents developing a more sophisticated functional thinking. Second, to implement the operative principle, one has to shift up or down on the vertical axis for one aspect of functional thinking and vary the task accordingly as described above.

The goal of the framework is to develop functional thinking in students' minds by guiding them to be engaged in an operative process. Consequently, the role of the FOM in the learning process needs to be discussed (see Fig. 2). As it is meant to be used for task design, its focus of application lies on the side of the task developer. It then affects the students in two ways: On the one hand, the students work through the different steps of a task sequence and by this process through different variations of the problem in the sense of the operative principle. We call this the operative process through the tasks. It might also be seen as an external requirement. By solving the task the students follow the variations which were set up through the task design while evaluating and reflecting on the changes. On the other hand, the students apply actions or operations within a task to objects and evaluate the effects of these actions with the question in mind, 'What happens with ... if ...'. Accordingly, we call this the operative process within the task. This might be seen as an internal requirement or strategy in the problem-solving process. This way of working is then explained again in the theoretical framework, especially with regard to functional thinking. 
Fig. 2 The role of the FOM in the learning process as an interaction between teachers, students and task is illustrated. Students work on tasks developed in accordance with the FOM in the sense of the operative principle, that is, by following the steps of a task sequence and by applying actions or operations within a task. This process is supported by the implementation of digital technologies

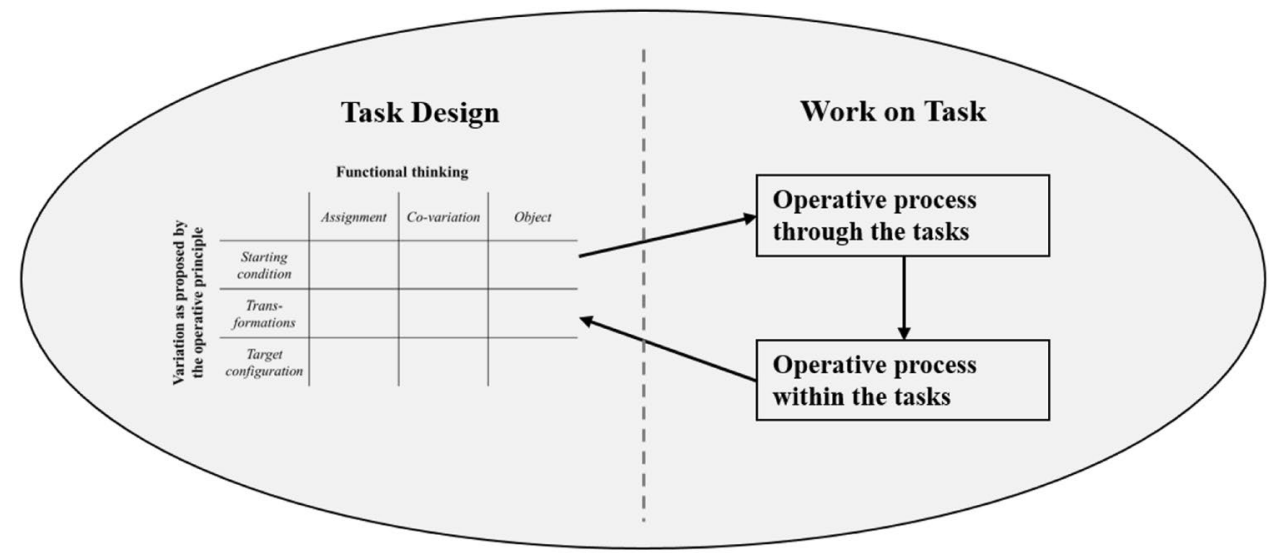

Digital technologies
The learning process as a whole, from the task development on the side of the task design to the work on the task by the students, is supported by the implementation of digital technologies.

An illustration of the FOM is provided by two examples of task sequences for Grade 8 of a German secondary school. Their curriculum initially focuses on functions especially dealing with linear functions. The tasks presented here are intended for deepening and consolidating their knowledge, which means students have already received an introduction to the related subject.

\subsection{Task 1: Linearity-Perimeters of regular polygons}

The starting point of this task is a square with the given side length as an independent and the perimeter of the square as a dependent variable, which is the target in the context of the FOM. The students should first construct a square with a slider (see Fig. 3) to make possible transformations accessible to them, which manipulates the side length (alternatively such a file can be made available). The first part of the task is to alter the starting condition and observe its impact on the perimeter. Hereby, the focus is on the aspect of assignment as the students investigate certain pairs of values; thus this translates to the cell starting conditionassignment of the FOM. Applying the first design principle to move right on the horizontal axis, we focus on the co-variation aspect next by observing by how much the perimeter changes if the side length changes by $1 \mathrm{~cm}$. (FOM: starting condition-co-variation).

A square $\mathrm{ABCD}$ is given with a side length of $2 \mathrm{~cm}$. Construct a square with the side length $a$, where $a$ can be modified by a slider.
1. Determine the perimeter for a side length of 2,3 and $5 \mathrm{~cm}$.

2. By how much does the perimeter of the square change if the side length increases by $1 \mathrm{~cm}$ ?

3. How much does the side length need to change to obtain a perimeter which is $6 \mathrm{~cm}$ longer? Does this depend on the initial side length of the square?

4. As a comparison, now consider a regular triangle and a regular hexagon. Which shape has the largest margin in slide length if each perimeter changes by the same amount? Justify your answer!

Concentrating further on this aspect, the task is modified with regard to target configuration-co-variation by now altering the perimeter instead of the side length. The question of whether this relies on the original side length of the square, motivates the consideration of posing an additional enquiry about the object-aspect. The fourth part of the task continues with a variation corresponding to the cell starting condition-object by asking students to examine a regular triangle and a regular hexagon. The developmental process of the task is illustrated by Fig. 4.

A dynamic representation is a powerful tool in this case, as the alteration of the sides can be realized, and so can the relationship between the length of the side and the perimeter when viewed as a whole by using the trace facility (see Fig. 3).

\subsection{Task 2.1: Adjusting the coordinate system- linear functions}

The initial starting point is the following task: Draw the graph of the function $f(x)=2 x+1$. Accordingly, a coordinate system and function equation are given in order to draw the corresponding line. In relation to the FOM, the coordinate system and function equation are the starting conditions, the drawn graph is the target configuration, and the 


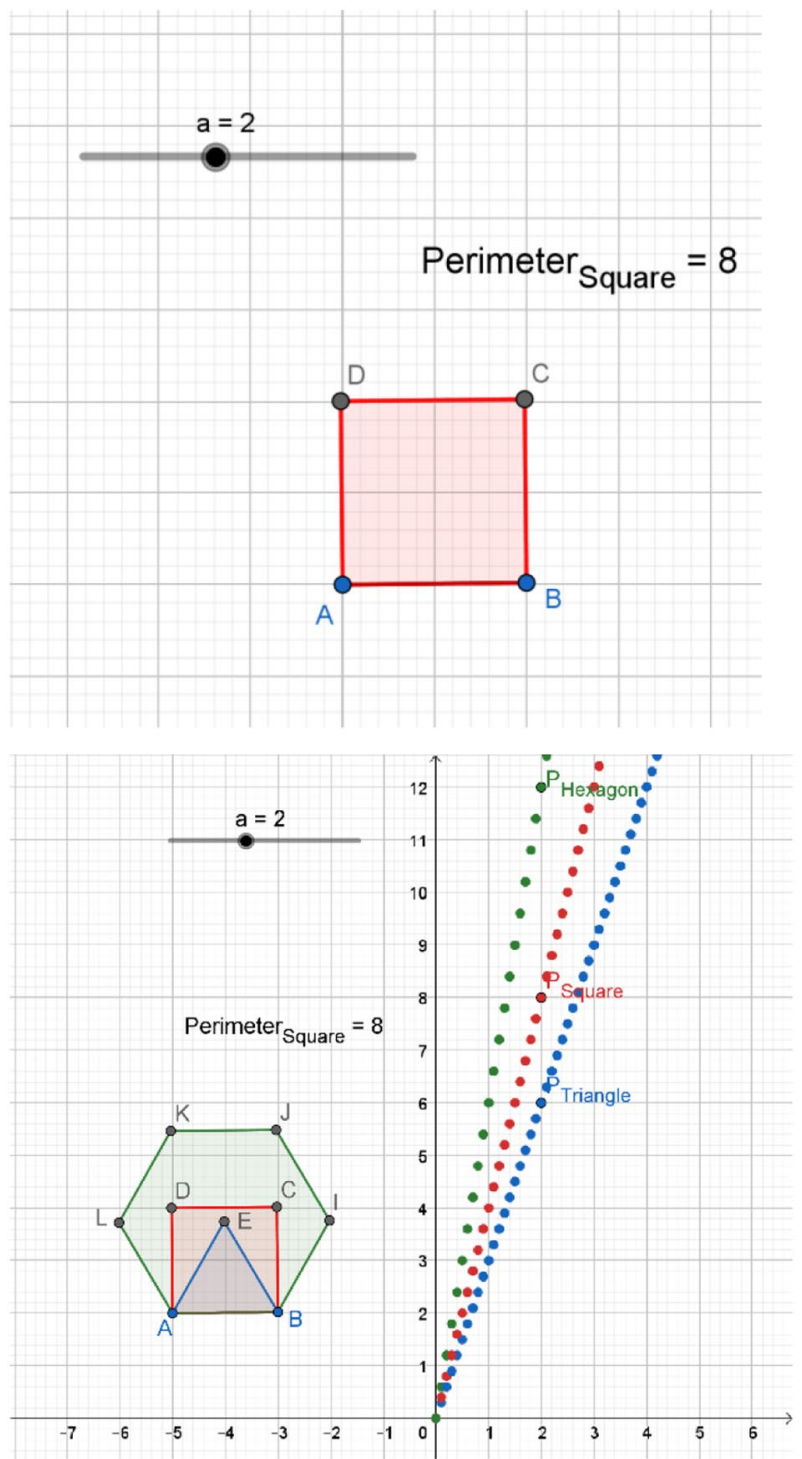

Fig. 3 Task sequence about the linearity of the perimeter of regular polygons possible ways to achieve this-such as drawing in the y-axis intercept or calculation of two points-are the transformations. One possible variation now, is to invert this. Instead of a coordinate system the line is given, and a suitable coordinate system needs to be drawn to represent the desired function equation (Herget 2017, p. 9). Even solving this with pen and paper poses an interesting and challenging task. The use of a digital learning environment (in this case GeoGebra), however, offers more possibilities: the coordinate system can be moved around in its entirety or rotated around its origin; and the axes can be compressed and stretched at will. Students can immediately observe the impact of their changes on the graph. In addition, they can display the resulting function term as a means of verification and feedback. Figure 5 shows an example of a solution as well as the task at hand.

1. A line is given. Adjust the coordinate system in such a way that the line represents the function $f$ $(x)=2 x+1$. Hint: The coordinate system can be adjusted by moving the blue dots.

2. Repeat the first task with the following restriction: Do not (a) rotate the coordinate system; and (b) stretch or compress the axes.

3. Give your neighbor a similar task!

With regard to the FOM, the first part of the task can be found in the cell starting condition-object, as by inverting it, the starting condition has, of course, completely changed. It corresponds to the object aspect since the line has to be seen as the graph of a function as a whole through reading properties from the graph and interpreting the impact when the coordinate system changes. Then the second design principle, namely, moving on the vertical axis, is applied as the task is modified according to the cell transformationsobject, by restricting the available operations. Depending on the solution strategy for (1), students should now adjust the coordinate system without rotating it or stretching/compressing the axes. Finally, the learners are encouraged to think of their own variations regarding the cell target configurationobject by giving each other similar tasks.

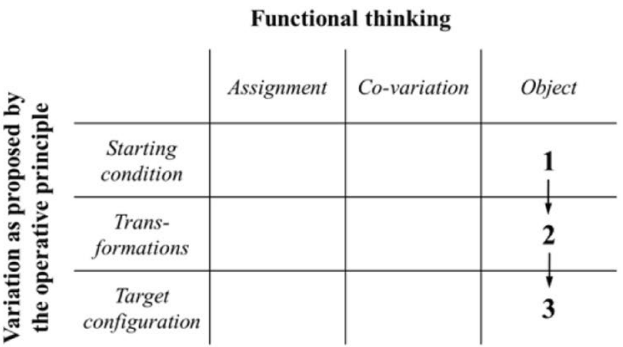

2 already starts with considering the object aspect. Further variations aim to deepen the operative process within the task
Fig. 4 Two examples of developmental processes through the variation of the task in relation to the FOM (le). Task 1 begins at the assignment aspect and moves stepwise to the object aspect (ri.) Task 


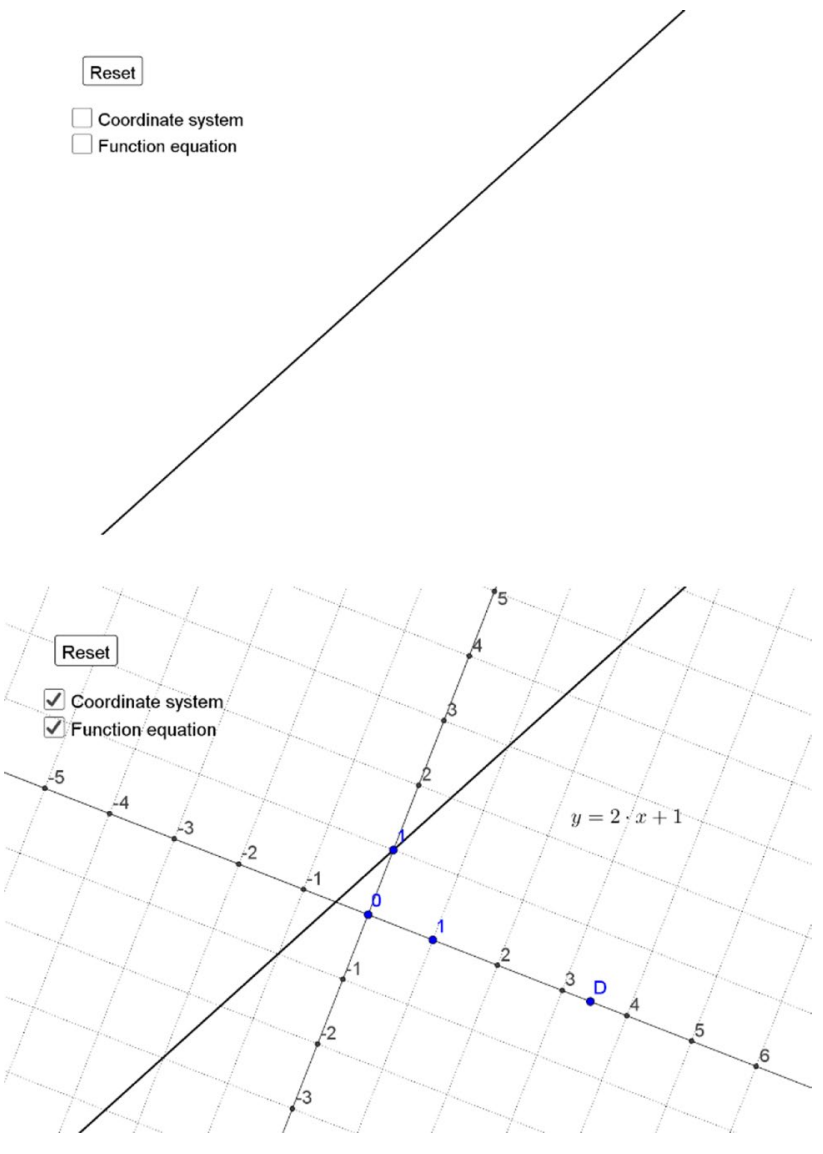

Fig. 5 Task sequence to draw the graph of a linear function. The coordinate system should be adjusted in such a way that it represents the line of the given function

The following tasks were used for the interviews. Here they are covered only very briefly.

\subsection{Task 2.2: Adjusting the coordinate system- inversely proportional function}

In analogy and continuation of task 2.1 we consider a hyperbola instead of a line. Therefore, there is a transition from a linear function to an inversely proportional function with $f$ $(x)=\frac{1}{x+a}+b$ which is covered in the same year of school. The same tools to adjust the coordinate system were made possible for the students, except for rotating it since it is not applicable in this situation, at least for students in the 8th grade. It can be classified in the FOM the same way as task 2.1 .

\subsection{Task 3: Graphs of linear functions}

The task here reads, 'State constraints for the linear function $f(x)=m x+b$ in such a way that it does not pass through the second quadrant' (FOM: target configuration-object). The task is altered according to the FOM and leads to the question, 'What are the parameter values, when observing a sum of two linear functions?'.

\subsection{Task 4. Transformations of graphs of linear functions}

The introductory request here is, 'Given the linear function $f$ $(x)=m x+b$, describe what impact $a$ has on the graph of the function $g(x)=a f(x)$ ' (FOM: starting condition-object). As a variation, the addition of a parameter (FOM: transformations-objects) $h(x)=f(x)+c$ and, where appropriate, a combination of multiplication and addition were discussed $k(x)=a(f(x)+c)(F O M:$ transformations-objects $)$.

\section{Methodology}

To answer the research questions 2 and 3 concerning the use of digital technologies and the students' functional thinking while working with the developed tasks, we conducted taskbased interviews. This was part of an evaluation study of the regular use of tablets starting in 8th grade at four German secondary schools (Gymnasium). The students attending the participating schools could choose, at the end of the 7th grade, whether they continued in a class using tablets or not. Two of the schools used iPads, one Android Tablets and one Microsoft Surface computers. The classes using tablets were monitored for the entire year of schooling for the purpose of the study.

\subsection{Participants and procedure}

This paper presents the result of the analysis of 12 taskbased interviews, 7 of which were conducted around midyear and 5 of which were conducted at the end of the school year. The students were between the age of 14 and 15 and represented the full range of mathematical ability in grade 8. In each case, two students were interviewed together to give them the opportunity to swap ideas about solving the task and to explain the solution to each other. Their teachers combined students into pairs with homogeneous achievement levels. One of the researchers conducted the interviews and provided guidance when needed, especially regarding the handling of GeoGebra. During both points in time of the study, the interview began with general questions regarding the use of the tablets and short introductory tasks with pen and paper to get into the topic. This was followed by the part of the interview relevant for the following analysis. Each group was asked to solve two tasks using the tablet, which recorded the activities of the students with a screencapture program. Additionally, the sessions were audiotaped 
Table 1 Interview tasks: For task 2.1 and 2.2 the students used the GeoGebra file provided to them, for task 3 and 4 they were able to use it as they saw fit

\begin{tabular}{ll}
\hline Mid-year & End-of-year \\
\hline $\begin{array}{l}\text { Task 2.1: Adjusting coordinate system; linear } \\
\text { function }\end{array}$ & Task 4: Influence of parameter on graph; linear function \\
$\begin{array}{l}\text { Task 3: Location graph of lin. function regarding } \\
\text { quadrants }\end{array}$ & $\begin{array}{c}\text { Task 2.2: Adjusting coordinate system; inversely pro- } \\
\text { portional function }\end{array}$ \\
\hline
\end{tabular}

and the combination of both audio and screen-capture was transcribed afterwards.

\subsection{Interview tasks}

As the 8th grade of the secondary school covers linear functions in the first half-year, the mid-year-interview first addressed the task of adjusting the coordinate system (task 2). Here, students were expected to adjust the coordinate system in such a way that the graph matches the function $f(x)=2 x-1$. Then, the second part of the task introduced the location of the graphs of linear functions in relation to the quadrants of the coordinate system (task 3). Here, students could access GeoGebra on the tablet as they saw fit.

The interviews at the end of the school year first covered task 4, an exercise about the influence of an additional parameter on a general linear function. Again, the students had unrestricted access to GeoGebra. The final task was task 2.2, which involved adjusting a coordinate system for an inversely proportional function. Table 1 provides an overview of the tasks dicussed in the interviews.

For both points in time of the study, the focus was on linear functions and developing functional thinking, with special focus on the object aspect, as the use of digital technologies seems especially promising for this otherwise difficult aspect. Furthermore, the assignment and co-variation aspect can be seen as prerequisites and thus can be expected to be observed in the students' actions as well. The plotting and the location of the graph of a linear function were chosen as a central theme for mid-year. At the end of the year, the more challenging tasks, which involved the transformation of graphs and the transition of the results of linear functions on the level of objects with inversely proportional functions, were discussed.

\subsection{Data analysis}

The interviews transcripts were analyzed in accordance with the structured content analysis (Kuckartz 2016). As the students attended different schools, it was expected that they would differ in their previous content-related knowledge and on a technical level with regard to the utilization of digital technologies. Therefore, we first checked if and how students were able to use their previously learned expertise for the solution of the tasks, since these were intended to deepen students' knowledge. Subsequently, we were interested in their handling of digital technologies and especially the utilization in the operative process (research question 2). Finally, we got to the actual goal, namely, which types of functional thinking appear while solving the tasks, and how the operative process can promote its development (research question 3). We evaluated both results of the mid-year and end-of-year interviews in comparison with each other. In this paper, we focus on tasks 2 and 4 for a more detailed analysis.

Table 2 Example of categories for the category system 'digital technologies (DT) usage'

\begin{tabular}{|c|c|c|c|}
\hline Category system & Categories & Description & Anchor examples \\
\hline \multirow[t]{6}{*}{ DT usage } & $\begin{array}{l}\text { Variation within the learning arrange- } \\
\text { ment }\end{array}$ & $\begin{array}{l}\text { The students use the tools given by the } \\
\text { learning arrangement }\end{array}$ & $\begin{array}{l}\text { [Moves coordinate system so that } \\
y \text {-intercept is correct] }\end{array}$ \\
\hline & $\begin{array}{l}\text { Feedback through the learning arrange- } \\
\text { ment }\end{array}$ & $\begin{array}{l}\text { The students use the feedback given by } \\
\text { the learning arrangement }\end{array}$ & $\begin{array}{l}\text { [The resulting function equation is } \\
\text { displayed] }\end{array}$ \\
\hline & Usage of sliders & $\begin{array}{l}\text { The students create or use sliders to } \\
\text { vary parameters }\end{array}$ & [They add sliders and vary a] \\
\hline & Creating objects & $\begin{array}{l}\text { The students create new objects, e.g. } \\
\text { lines }\end{array}$ & $\begin{array}{l}\text { [They enter } 2 x+1 \text { and get the corre- } \\
\text { sponding function graph] }\end{array}$ \\
\hline & Adjusting existing objects & $\begin{array}{l}\text { The students adjust existing objects, e.g. } \\
\text { by modifying the function equation } \\
\text { of a line }\end{array}$ & $\begin{array}{l}\text { [They change } g(x) \text { accordingly, that there } \\
\text { is }+\mathrm{a} \text { ] }\end{array}$ \\
\hline & Usage of zoom & $\begin{array}{l}\text { The students zoom into the graphing } \\
\text { area }\end{array}$ & $\begin{array}{l}\text { [Zooms in to check if the } y \text {-axis intercept } \\
\text { is at 2.5] }\end{array}$ \\
\hline
\end{tabular}


Accordingly, the main category systems for the analysis were previous knowledge, utilization of digital technologies, functional thinking and operative process. The categories for functional thinking, namely, assignment, covariation and object—as well as the ones for the operative process, namely, operative process through the task, operative process within the task and evaluation/reflection-were deduced from the theoretical background. The categories of the other two systems, however, were inductively generated from interview transcripts in accordance with the structured content analysis (Kuckartz 2016). As an example, the category system for digital technologies usage is given (cf. Table 2).

\section{Findings}

The presentation of the results of the interviews is structured regarding the above-mentioned main categories and research questions. The interviews were numbered chronological from 1 to 12, so S9-1 stands for Student number 1 of interview number 9. An additional analysis of two students' sheets of task 2.1 of the mid-year interview can be found in the paper by Günster (2019).

\subsection{Applying prior knowledge}

By mid-year the students were able to adjust the coordinate system and thereby draw a graph using the procedures familiar to them (task 2.1). Most of the students firstly selected the y-intercept and then located a second point with the slope triangle, or determined the slope of the line. Typical errors arose such as mixing up $\Delta y$ and $\Delta x$ (Nitsch 2015), which they in most cases rectified with the aid of the feedback of the learning environment by displaying their current graph as a function equation and adjusting it accordingly. Alternatively, they calculated two points using the function equation and matched the coordinate system so that the line ran through those points.

At the end of the school year, most students described the meaning of the slope and the y-intercept with regard to the location of the graph without difficulty (task 4). However, they showed no prior knowledge on the impact of another parameter on the graph as this is a transfer task. At this point, they could now engage in an operative process with the support of digital technologies, which we describe in the next section.

Furthermore, the students showed difficulties with the task regarding the hyperbola (Task 2.2) in interpreting the function equation-in this case $f(x)=1 /(x+2)$-correctly, and in particular, in grasping the connection between the zero of the denominator and the asymptote of the graph. For example, S11-1 correctly realized that -2 is a gap in the domain. The following shift, however, shows an insufficient distinction of the terms y-intercept and asymptote (cf. Fig. 6).
Fig. 6 Example of students' solution for Task 2.2: $f(x)=1 /$ $(x+2)$. They mixed up the meanings of an asymptote and the y-intercept as they know it from linear functions

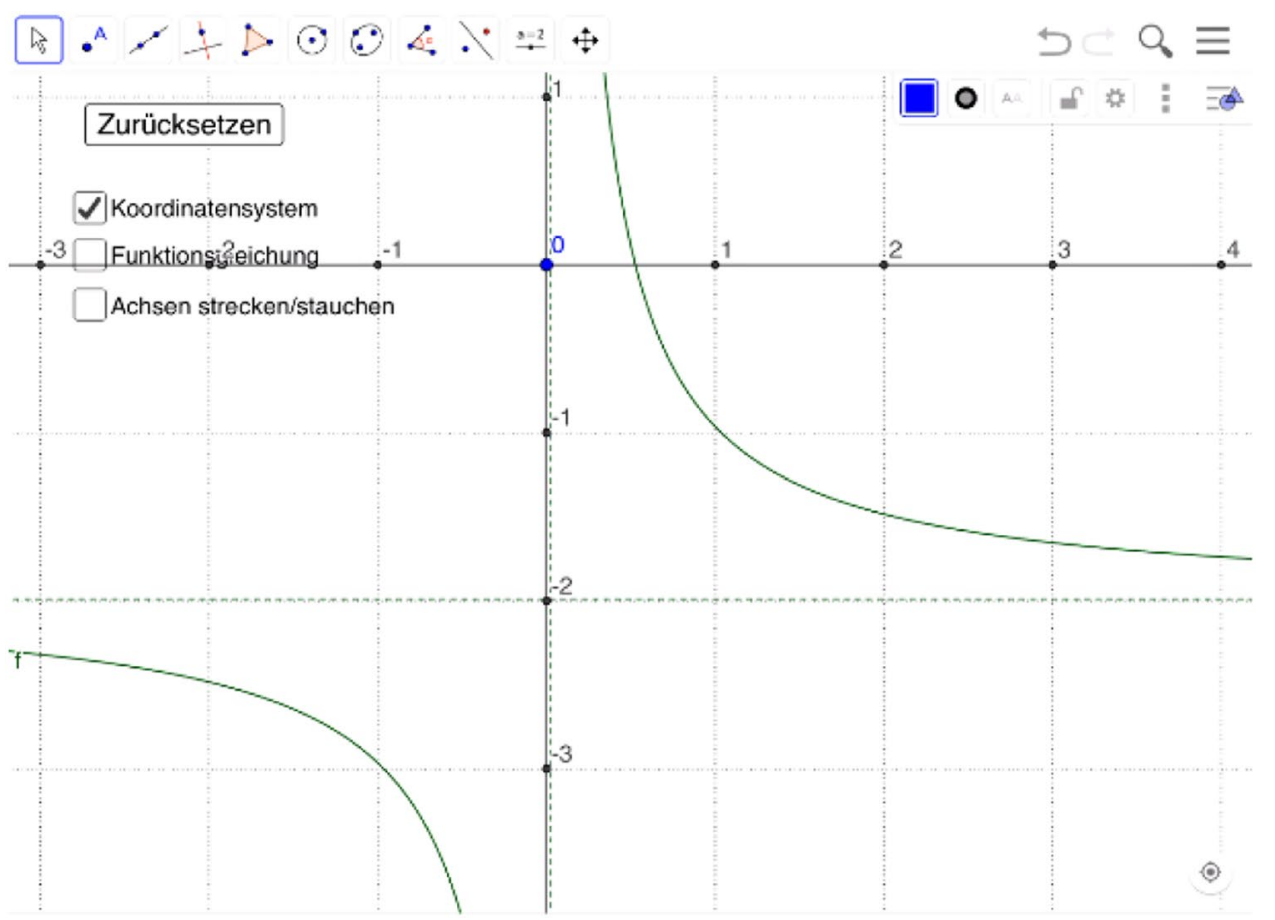


Int: What does $x+2$ in the denominator mean?

S11-2: $x$ refers to the $x$-axis I would say and then 2 from the $y$-intercept

$[\ldots]$

S11-1: yes, -2 cannot be put in, that means

S11-2: the asymptote has to be at -2, right? Like this? [Moves coordinate system in such a way that -2 is a horizontal asymptote] Or not?

The first answer hints to a misconception to what a function means, namely a relationship between two variables (cf. assignment aspect), while evidencing prior knowledge about the $y$-axis intercept in the case of linear functions. The student had thus not realized the importance of the variable $x$ being in the denominator. While S11-1 recognized this and rightfully stated the restriction to the domain, S11-2 mixed up the notion of asymptote and y-intercept as he was still trying to apply the accustomed routine of shifting the graph in the horizontal direction known from linear functions. Here, too, the learners could utilize the feedback of the learning environment in a useful manner for improvement, by displaying the function equation resulting from their shift and correcting their solution.

\subsection{Utilizing digital technologies}

Regarding the use of digital technologies, there are vast differences between students. First, a distinction must be made between the tasks: The tasks, which involve adjusting the coordinate system (Task 2), only provide the possibility of changing the representation by moving the dots or activating feedback. To investigate the influence of parameters, however, the learners could access the entirety of GeoGebra as a fully functional tool, and for this purpose they had to first create their own environment in order to engage in an operative process. We first focus on how they dealt with both possibilities, before describing how the students utilized digital technologies in the sense of the operative principle.

\subsubsection{Technical skills with digital technologies}

When adjusting the coordinate system, students had no problems using the given operations-shifting, rotation, stretching and shrinking. They utilized them without preference as, for example, in modifying the slope by rotation or by stretching/shrinking the axes.

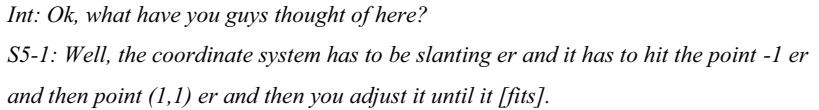

In this case, the student uses prior knowledge in that the line has to run through two points. While exploiting only the rotate and shift option, they are able to adjust the coordinate system to be slanting the right way in a stepwise manner by switching between both tools multiple times.

Around mid-year, with free access to a tablet, some students had no idea on how to use GeoGebra to solve the task.
Fig. 7 The students created three sliders to explore the influence of a parameter, which is multiplied with the function equation, graphically. Two sliders ( $m$ and $t$ ) change the slope and the y-intercept, the third determines the value of the parameter

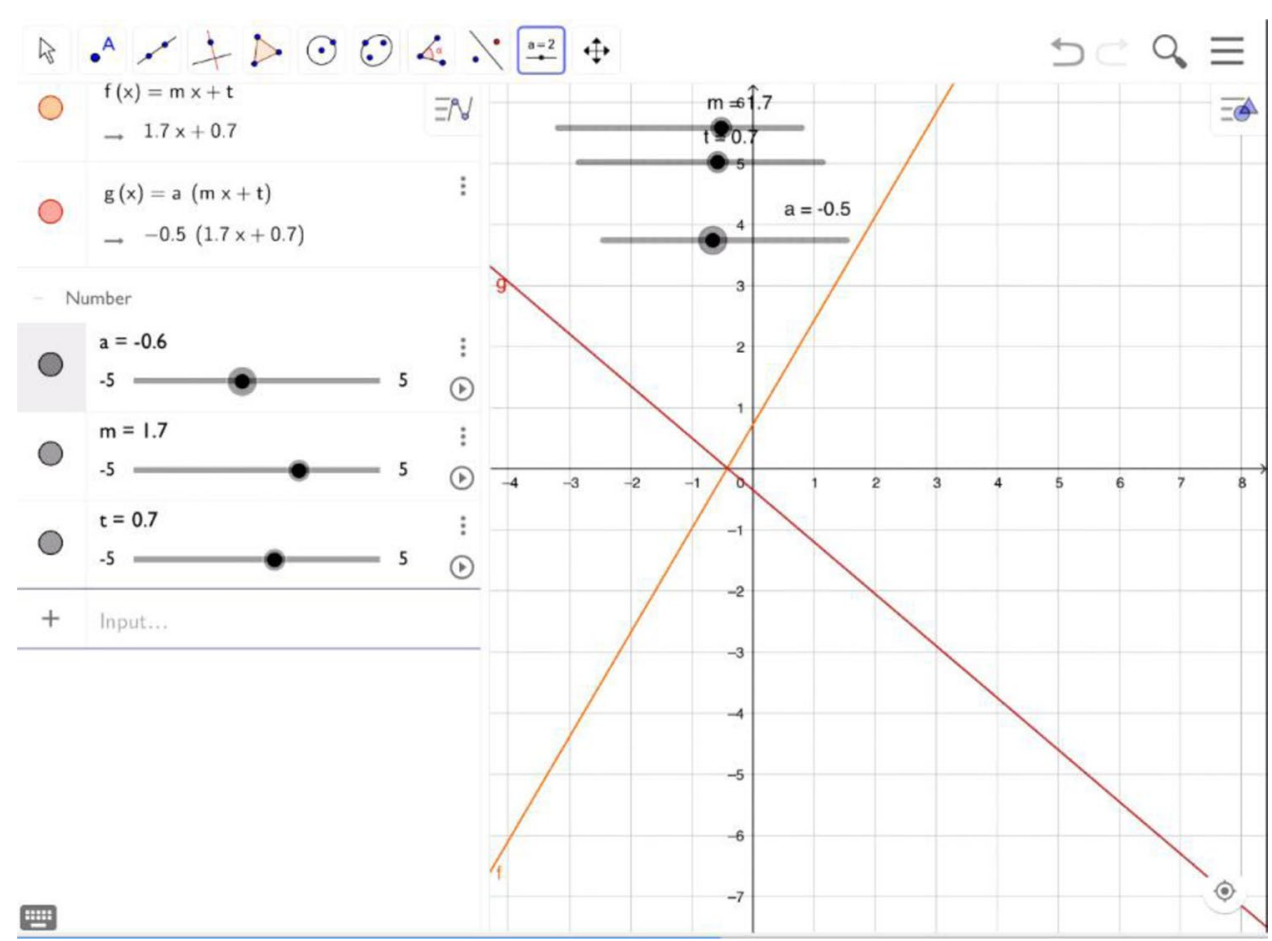


Some entered specific functions as a crosscheck and used the tablet as a function plotter. They were partially able to modify their functions systematically, by, for example, viewing a negative slope instead of the positive one with the same absolute value and observing the changes. There were those who were using GeoGebra intensively as a tool by mid-year, as well. As an example, when asked what the sum of two linear functions is, they created an environment with four individual sliders, of their own accord.

The interviews at the end of the school year demonstrated a similar picture. While a few students began to create sliders by starting with the introductory question about the influence of the slope and the y-intercept on the graph (Task 4) to once again test their impact (cf. Fig. 7), others still had difficulties just plotting a function and needed a lot of assistance from the interviewer to create an appropriate learning environment. Nevertheless, all learners were able to use the learning environment, whether created with help or not, for investigating during the rest of the interview. However, it seemed as if the less experienced tended to let the sliders shift in an automated manor, while the experts changed them in a systematic way by changing only one slider at a time, for specific values of the other ones for example.

\subsubsection{Role of digital technologies for the operative principle}

We distinguished two ways of applying the operative principle, namely, the operative process through and within the tasks. Therefore, we address the role of digital technologies for both of these and begin with the variation within the tasks through the students.

When we described how the students dealt with digital technologies in a learning arrangement, we gave an example where the students were adjusting the coordinate system in a stepwise process (see first interview excerpt). This represents an operative process within the tasks since each time students finished an action they evaluated the change it caused to the relationship between the line and the coordinate system and adjusted their following action accordingly. Feedback by the digital technologies, by showing the function equation that matched their adjustments of the coordinate system, supported their evaluation of the effects of their actions as well as revealing, in some cases, misconceptions.

In the case of using GeoGebra as a tool, some students plotted specific functions and then adjusted them by changing the sign or the absolute value of the slope. Thus, it seemed that students had checked whether the changes to the representation they had performed mentally were correct. This implies that the digital technologies supported them in their operative process thus as a feedback tool. All students created sliders during the interviews - some with the help of the interviewer-to assess the influence of additional parameters on the graph of a linear function. They were then able to apply the action of multiplying or adding a parameter to the equation of a linear function through the variation of the sliders. Students viewed the effects of those actions and evaluated them. As a result, many were able to describe the changes of the function with the graphical representation"the line rotates around zero"-but had difficulties interpreting this within the context and describing their reasoning. We discuss this point further when we outline the analysis regarding the aspects of functional thinking.

Digital technologies also supported the variation of the tasks through the design with the FOM. First, the task sequence around the adjustable coordinate system would not be possible in this way without an implementation with the aid of GeoGebra. By varying the task further-in this case through the restriction of the tools they were allowed to use-students were encouraged to use all available actions and view their effects. Otherwise, students would simply use the method that led them to the first solution and stop work as it does not seems necessary for them to look for further possibilities. For the other tasks, the use of digital technologies seems to support the transition between the different variations, and thereby, parts of the tasks. For example, in Task 4 , when considering the influence of an additional parameter on the graph of a function, digital technologies supported students' progress from the action of multiplying the function equation with a certain parameter to adding it, as only a change in the sign in front of the parameter was needed. This seemed to make the evaluation of variances and invariances between the two variations of the problem more accessible and thereby assisted the operative process through the tasks.

\subsection{Functional thinking}

The analysis concerning the students' functional thinking is structured according to the distinction between the tasks in two respects, namely, adjusting the coordinate system through a given learning arrangement, and concerning the influence of additional parameters.

\subsubsection{Adjusting the coordinate system}

As stated before, the students used their prior knowledge for this task by calculating points, reading them from the graph, or by arguing with the aid of the slope triangle what should be allocated to the assignment and covariation aspect. There were few signs, however, that they viewed the line with the coordinate system as a whole regarding the object aspect. To the question of what changed by stretching or shrinking the $\mathrm{x}$-axis, one group responded in the following way: 


$\begin{array}{ll}\text { S1-1: } & \text { The grid boxes got larger, when one was at } 1 \text { the boxes got like } \\ & \text { longer. } \\ \text { Int: } & \text { When we are talking about linear functions, did the y-axis } \\ & \text { intercept change? Did the slope change? } \\ \text { S1-1: } & \text { No, because it's still } 1 \text { to the right and } 2 \text { up. } \\ \text { Int: } & \text { And regarding this line, which is here... what changes when one } \\ & \text { varies the } x \text {-axis? Does the y-axis intercept change? } \\ & \text { [Students are shrinking and stretching the } x \text {-axis.] } \\ \text { S1-1: } & \text { Mhm. No. } \\ \text { Int: } & \text { What does change, though? } \\ \text { S1-2: } & \text { Actually, it is only getting more precise. }\end{array}$

In the first response, students just described the change of the graphical representation without considering the relationship of the coordinate system and the graph. Here, there seems to be a lack of connection between the graphical and symbolic representations of a function. This is displayed further when the student was asked about the effect on the characteristic parameters of a linear function, since they saw no change but stated the value of the slope given by the function equation. The final response goes back to the graphical representation as they commented that the change of the size of the boxes was getting more precise. Students appeared to associate this change with what they discovered by zooming into an object.

The task of adjusting the coordinate system given a hyperbola instead of a linear function caused major difficulties for the students due to their lack of prior knowledge. Nevertheless, some groups showed their understanding of shifting graphs, based on an added parameter, by applying it to this situation in order to adjust the coordinate system to the function equation $f(x)=1 / x-1$. For this purpose, they dealt with the object aspect by transferring their experiences with the object 'line' to the object 'hyperbola' and using these to solve the problem. They knew, for example, that a change in the y-intercept of the graph of a linear function results in a horizontal movement in relation to the coordinate system. The ' -1 ' in the function equation of the hyperbola represents a similar action and hence means that the whole graph has to be shifted down by -1 in relation to the coordinate system. They are thus able to view the graph as an object and apply this action to it.

\subsubsection{Impact of parameters on linear functions}

For this task, students should first describe the influence of a parameter, which is multiplied by the entire function concerning the object aspect. To analyze this situation, all groups were required to represent this in GeoGebra. This led to a similar difficulty - making a connection between effects of the action on the function in the graphical representation and its interpretation in the symbolic one.
With further exploration, students determined whether the y-intercept or gradient changed, but did not relate this to the function equation itself (see for example this section of an interview).

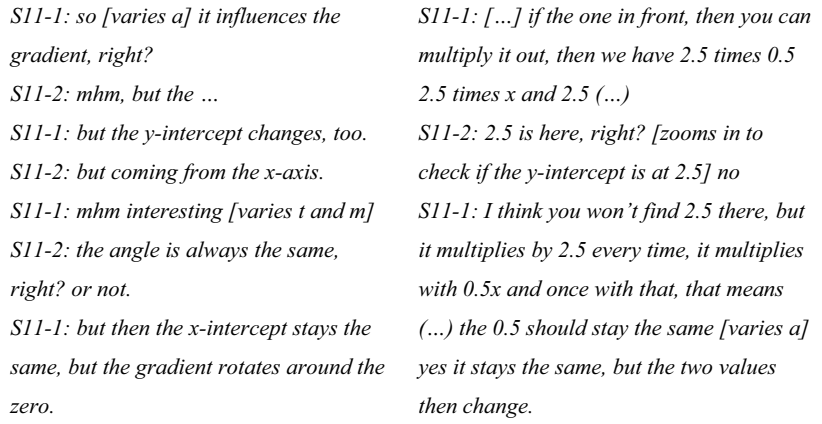

On the left, the students showed evidence of their understanding of the graph of the function as a whole (object aspect) as they applied different actions to it by varying the sliders and relating the changes of the graphical representation back to the characteristic concepts of a linear function. In this operative process they realized that the zero- - the $\mathrm{x}$-intercept-stays the same while the other parameters change. As students proceeded to try to relate this to the function equation, they find certain values of the formula represented in the graph - in this case 2.5 , the value of the parameter by which the function equation is multiplied. However, they concluded that this was not the case, since the slope and the y-intercept, which can be read off the graph, are multiplied by it.

In the second part, the addition instead of multiplication of a parameter was the action the students should investigate. Here it was much easier for the students to describe the changes with the help of the graphical representation and connect them to the symbolic representations regarding the object aspect, because the impact of adding another parameter results in similar changes to the graph as modifying the $y$-intercept. Only one group could handle the combination of both, namely, multiplying by a parameter and adding another. They again utilized the graphical representation with the aid of digital technologies to explore the situation and could describe qualitatively the influence of both parameters on each other; they operated with the function as an object. (cf. Fig. 8 and the section of the interview).

S11-2: $\quad$ and the smaller $a$, the larger $c$ and the smaller the $y$-intercept and if you make c smaller then the y-intercept is larger [changes $a$ and $c$ accordingly]

S11-1: $\quad$ that means if a becomes negative, it does the exact opposite.

Int: $\quad$ and what happens when I am close to zero for example at 0.3 ?

S11-1: $\quad$ it moves less [a at 0.3 and varies c]

S11-2: $\quad$ yes, it's on a smaller range 
Fig. 8 The students investigate how the variation of the parameters interact with each other when a function is multiplied by one and another one is added

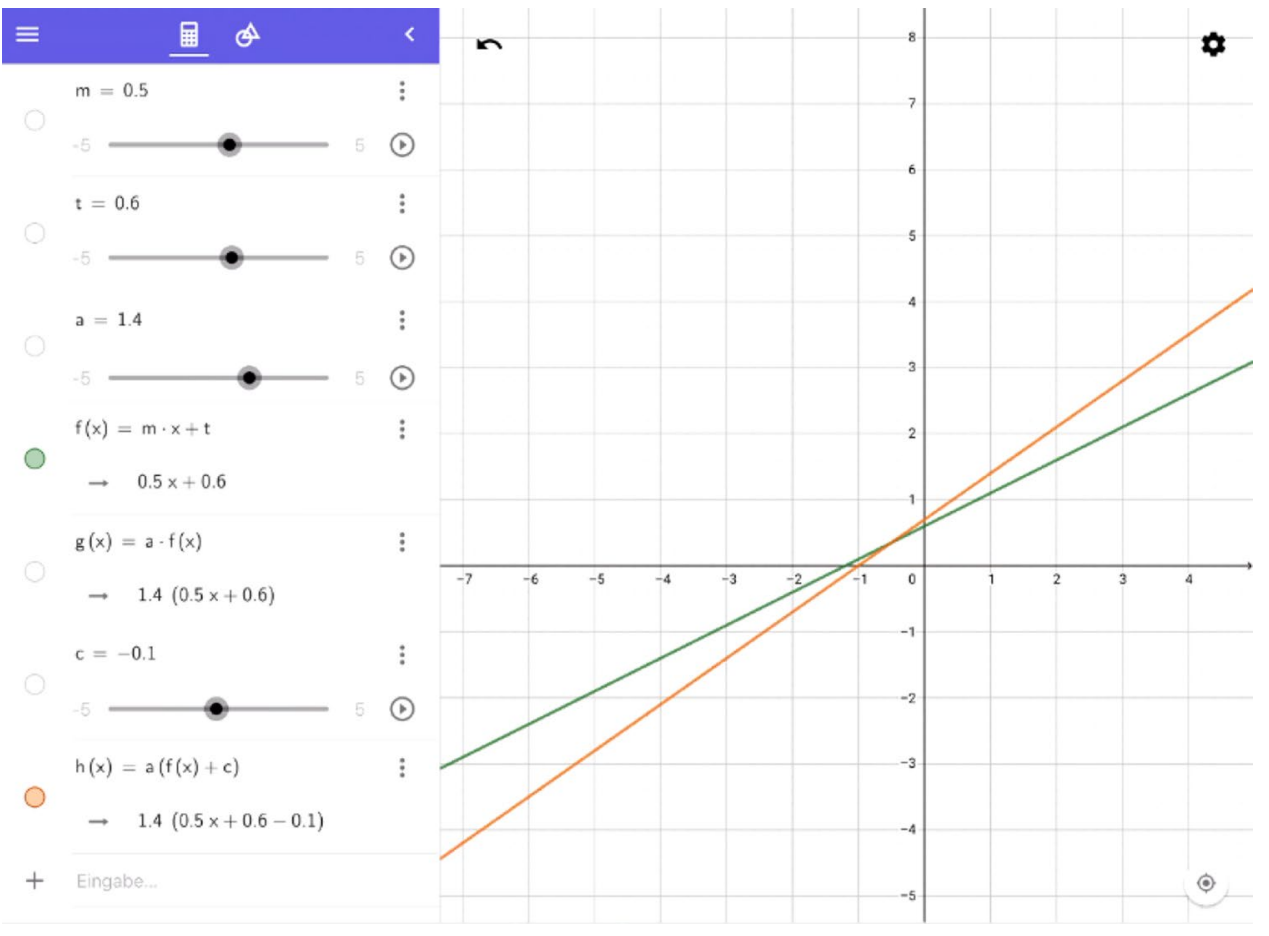

interviews in which they worked on tasks created in accordance with the FOM. The FOM and the deduced design principles seem to provide fitting guidance to construct problems and tasks for initiating students' actions related to variations, in the sense of the operative principle-variation of the starting condition, the transformations and the target configuration. In the presented study, the researcher used the FOM as a guideline to create tasks in the sense of the operative principle. A subsequent step could be the application of the FOM by teachers, as they would naturally be better able to assess the learning needs of their students and adapt the tasks accordingly.

Interviews with the students attending 8th grade provided insight, on a qualitative basis, into their activity when working with the tasks. The results showed that the tasks acted as intended and could be described according to Fig. 2 . On the one hand, students executed the operative process through the task by working through its sequences and variations according to the FOM. Thus, students were externally guided by the variations of the problem and they were able to view and investigate the changes those variations generated. On the other hand, they used the operative process within the tasks, for example, by creating sliders for parameters, thereby varying parameters of functions or rotating the coordinate system and evaluating the results. The students were internally driven to vary situations-in the sense of the operative principle - and reflect upon the results of the variations. In particular, the last step contributed to the development of functional thinking, depending on the problem, in its three aspects. However, we can only interpret the

Its application was illustrated on the one side by examples of tasks, and on the other side by an analysis of student 
resulting actions and explanations, because reflections and other mental processes are naturally not directly observable. Moreover, it is not possible to make statements about the long-term development of functional thinking based on the results presented here.

\subsection{Technical skills and role of digital technologies for the operative principle}

Barzel et al. (2006) observed, "given a tool, the genesis of a fruitful instrument is far from self-evident, but is the result of a social process, guided by a set of tasks in a given institution" (p. 929). This statement from 2006 was apparent in the presented interviews almost 15 years later. Although every student had access to a tablet throughout the entire school year, they developed very different skill sets when handling digital technologies. While some had problems entering a function equation, others used the tablet as a tool, which means that they made use of the options presented by the applications such as sliders and could use the commands of GeoGebra adequately in the situation. This poses the question for future teaching of how-next to the undoubtedly central development of understanding mathematics (here functional thinking) the proficiency in using these tools could be schooled in such a way that digital technologies could be applied for the desired purpose.

One option would be to define and construct the learning environment for the students more explicitly. This means, for example, that for Task 4, investigating the influence of the parameters, students could be provided with a ready-made file containing the corresponding objects, i.e., sliders and defined functions. However, it is necessary to be careful that the activities with such a provided file do not exhaust themselves in unsystematic experimenting without seeing the relationship between the representations and the mathematical objects 'behind'. Presenting 'baked' or 'half-baked' files (Kynigos 2007) hence has to be weighed up against students being expected to create everything for themselves, with the risk of failing at the first hurdle or spending too much time on the development of the supporting infrastructure. The creation of the task must therefore be matched to the goal of the lesson and adopted to the particular learning group.

The different levels of prior knowledge also poses a limitation to this study. Although we took this aspect into account in our analysis, the influence of prior knowledgealso with regard to the mathematical content-on the students' work on the tasks cannot be conclusively clarified. Furthermore, while we investigated the students' actions through screen capture, it could be beneficial to be able to analyse additional gestures, typical for the use of mobile devices like zooming in, via videography.
The sample tasks and the results of the interviews also show the potential of digital technologies for actions in the sense of the operative principle but also for task development with the FOM. Digital technologies made the evaluation of and reflection on actions, like shrinking/stretching the axis or multiplying a function by a parameter in the graphical representation, possible. The operative process through the tasks became more accessible, as changes between tasks could be implemented and compared easily. Digital technologies allowed students to work within the notion of the operative principle, to engage in operative processes within the tasks, and directly to experience and interpret the consequences of their actions.

\subsection{Functional thinking and the operative principle}

The goal of the FOM and the generated tasks was the promotion of functional thinking. In the interviews, we focused on the object aspect, as the assignment and covariation aspect can be seen as prerequisites and the object aspect seems especially suitable for the use of digital technologies through acting on the functions directly. In working with functions as objects two possibilities can be distinguished, i.e., manipulating and reflecting (vom Hofe 2004). While manipulation focuses on the function as an object in an encapsulated form that can be changed, reflection takes the object out of its case, and makes connections between various forms of representations in order to emphasize the underlying functional relation. The presented tasks dealt with manipulating the functions, but encouraged reflection as well. They gave an opportunity to inspect the aspects of functional thinking from different points of view, investigate variances and invariances and therefore promote an operative process.

The results of the interviews showed that learners have difficulties relating the changes based on manipulating the graphical representation to the symbolic representation. This is well known from many empirical studies. In this study, these difficulties should be counteracted on the one hand by the successive variation of the problem in the sense of the operative principle. On the other hand, students are engaged in an operative process through the assistance of digital technologies as they combine both levels of representation by making the actions clear with the graph and the underlying function equation. Samples from the interviews exemplified the success of this method, e.g., by successively adjusting the coordinate system or by varying the parameters with the sliders and evaluating the resulting changes in both representations. However, the importance of the subsequent reflection process must be emphasized again; without it, the manipulation remains on an observation level and does not evoke functional thinking. 
In summary, the theoretical framework through the FOM seems to offer a constructive way to utilize the potential of digital technologies for the development of functional thinking. As students' interviews provided insight, on a qualitative basis, into how they dealt with the developed tasks and the resulting reflection processes with respect to their functional thinking, further studies need to investigate improvement in understanding on a quantitative level.

Acknowledgments Open Access funding provided by Projekt DEAL.

Open Access This article is licensed under a Creative Commons Attribution 4.0 International License, which permits use, sharing, adaptation, distribution and reproduction in any medium or format, as long as you give appropriate credit to the original author(s) and the source, provide a link to the Creative Commons licence, and indicate if changes were made. The images or other third party material in this article are included in the article's Creative Commons licence, unless indicated otherwise in a credit line to the material. If material is not included in the article's Creative Commons licence and your intended use is not permitted by statutory regulation or exceeds the permitted use, you will need to obtain permission directly from the copyright holder. To view a copy of this licence, visit http://creativecommons.org/licenses/by/4.0/.

\section{References}

Acevedo Nistal, A., van Dooren, W., \& Verschaffel, L. (2012). What counts as a flexible representational choice? An evaluation of students' representational choices to solve linear function problems. Instructional Science, 40(6), 999-1019.

Arnon, I., Cottrill, J., Dubinsky, E., Oktaç, A., Fuentes, S. R., Trigueros, M., et al. (2014). APOS theory: A framework for research and curriculum development in mathematics education. New York: Springer Science \& Business Media.

Barzel, B., Drijvers, P., Maschietto, M., \& Trouche, L. (2006). Tools and technologies in mathematical didactics. In M. Bosch (Ed.), European Research in Mathematics Education IV: proceedings of the fourth congress of the European Society for Research in Mathematics Education (CERME 4) (pp. 927-938), FUNDEMI IQS - Universitat Ramon Llull and ERME, Sant Feliu de Guíxols, Spain, 17-21 February 2005.

Bruder, R. (2000). Eine akzentuierte Aufgabenauswahl und Vermitteln heuristischer Erfahrung-Wege zu einem anspruchsvollen. Mathematikunterricht für alle Mathematik lehren und lernen nach TIMSS-Anregungen für die Sekundarstufen. Berlin: Volk und Wissen.

Burrill, G. (2017). Designing interactive dynamic technology activities to support the development of conceptual understanding. In L. Baccaglini-Frank (Ed.), Digital technologies in designing mathematics (pp. 303-328). Cham: Springer.

Churchhouse, R. F. (Ed.). (1986). The influence of computers and informatics on mathematics and its teaching. ICMI study series. Cambridge: University Press.

Core-PlusMathematics Project. (2015). Core-plus mathematics, course 1, student materials. New York: McGraw Hill Education.

Doorman, M., Drijvers, P., Gravemeijer, K., Boon, P., \& Reed, H. (2012). Tool use and the development of the function concept: From repeated calculations to functional thinking. International Journal of Science and Mathematics Education, 10(6), 1243-1267.
Drijvers, P., Doorman, M., Boon, P., \& van Gisbergen, S. (2010). Instrumental orchestration: Theory and practice. In V. DurandGuerrier, S. Soury-Lavergne, \& F. Arzarello (Eds.), Proceedings of the Sixth Congress of the European Society for Research in Mathematics Education (CERME 6), Institut National de Recherche Pédagogique and ERME, Lyon, France, 28 January-1 February 2009 (pp. 1349-1358).

Dubinsky, E., \& Harel, G. (1992). The nature of the process conception of function. The Concept of Function: Aspects of Epistemology and Pedagogy., 25, 85-106.

Flewelling, G., \& Higginson, W. (2003). Teaching with rich learning tasks: A handbook. Adelaide: AAMT Inc.

Giest, H., \& Lompscher, J. (2006). Lerntätigkeit-Lernen aus kulturhistorischer Perspektive. Ein Beitrag zur Entwicklung einer neuen Lernkultur im Unterricht (p. 2006). Berlin: Lehmanns Media.

Günster, S. M. (2019). Utilizing dynamic representations to foster functional thinking. In U. T. Jankvist, M. Van den Heuvel-Panhuizen, $\&$ M. Veldhuis (Eds.), Proceedings of the Eleventh Congress of the European Society for Research in Mathematics Education (CERME11, February 6-10, 2019). Utrecht, the Netherlands: Freudenthal Group \& Freudenthal Institute, Utrecht University and ERME.

Gutiérrez, A., Laborde, C., Noss, R., \& Rakov, S. (1999). Tools and technologies. European Research in Mathematics Education, 1, 183-188.

Herget, W. (2017). Aufgaben formulieren (lassen): Weglassen und Weg lassen—das ist (k)eine Kunst. Mathematik Lehren, 2017(200), $7-10$.

Hoyles, C., \& Lagrange, J.-B. (Eds.). (2010). Mathematics education and technology: Rethinking the terrain. The 17th ICMI study. New York: Springer.

Krüger, K. (2019). Functional thinking: The history of a didactical principle. In H.-G. Weigand, W. McCallum, M. Menghini, M. Neubrand, \& G. Schubring (Eds.), The legacy of Felix Klein (pp. 35-53). Cham: Springer.

Kuckartz, U. (2016). Qualitative Inhaltsanalyse: Methoden, Praxis, Computerunterstützung (3., überarbeitete Auflage). Grundlagentexte Methoden. Basel: Beltz Juventa.

Kynigos, C. (2007). Using half-baked microworlds to challenge teacher educators' knowing. International Journal of Computers for Mathematical Learning, 12, 87-111.

Leuders, T. (2015). Aufgaben in Forschung und Praxis. In R. Bruder, L. Hefendehl-Hebeker, B. Schmidt-Thieme, \& H.-G. Weigand (Eds.), Handbuch der Mathematikdidaktik (pp. 435-460). Berlin: Springer.

Leung, A., \& Baccaglini-Frank, A. (Eds.). (2017). Digital technologies in designing mathematics education tasks. Cham: Springer.

Leung, A., \& Bolite-Frant, J. (2015). Designing mathematics tasks: The role of tools. In H. Watson \& M. Ohtani (Eds.), Task design in mathematics education (pp. 191-225). Cham: Springer.

Nitsch, R. (2015). Diagnose von Lernschwierigkeiten im Bereich funktionaler Zusammenhänge: Eine Studie zu typischen Fehlermustern bei Darstellungswechseln. Wiesbaden: Springer Fachmedien Wiesbaden.

Piaget, J. (1967). Psychologie der Intelligenz (3rd ed.). Stuttgart: Rascher Verlag.

Trgalová, J., Clark-Wilson, A., \& Weigand, H.-G. (2018). Technology and resources in mathematics education. In T. Dreyfus, M. Artigue, D. Potari, S. Prediger, \& K. Ruthven (Eds.), Developing research in mathematics education: Twenty years of communication, cooperation and collaboration in Europe (pp. 142-161). Berlin: Springer.

Trouche, L. (2004). Managing the complexity of human/machine interactions in computerized learning environments: Guiding students' command process through instrumental orchestrations. 
International Journal of Computers for Mathematics Learning, 9(3), 281-307.

Vollrath, H.-J. (1989). Funktionales Denken. Journal für MathematikDidaktik, 10(1), 3-37.

vom Hofe, R. (2004). "Jetzt müssen wir das Ding noch stauchen!" Über den manipulierenden und reflektierenden Umgang mit Funktionen. Der Mathematikunterricht, 50(6), 46-56.

Vygotsky, L. S. (1978). Mind in society: The development of higher psychological processes. Cambridge: Harvard Business Press.

Watson, A., \& Ohtani, M. (Eds.). (2015). Task design in mathematics education. ICMI study 22. Berlin: Springer.
Wittmann, E. C. (1985). Objekte-Operationen-Wirkungen: Das operative Prinzip in der Mathematikdidaktik. Mathematik Lehren, 1985(11), 7-11.

Publisher's Note Springer Nature remains neutral with regard to jurisdictional claims in published maps and institutional affiliations. 